\title{
Summary of Case 5 from Sixth Drag Prediction Workshop: Coupled Aerostructural Simulation
}

\author{
Stefan Keye* \\ DLR, German Aerospace Center, 38108 Braunschweig, Germany \\ and \\ Dimitri Mavriplis \\ University of Wyoming, Laramie, Wyomin 82071 \\ DOI: $10.2514 / 1 . C 034427$
}

\begin{abstract}
A summary of participants' results for the coupled aerostructural simulation test case from the Sixth AIAA Computational Fluid Dynamics Drag Prediction Workshop held in Washington, DC, 16-17 June 2016, is presented. The test case focuses on a static aeroelastic simulation of NASA's Common Research Model civil transport aircraft in the wing/body configuration (i.e., without engines, pylons, and empennage). Flow conditions include the design point lift coefficient $C_{L}=0.5 \pm 0.0001$ at cruise flight. Aeroelastic simulations are performed on the "medium" baseline grid, coupled to a computational structural analysis, using either an existing finite element model or modal data. Experimental validation data for this test case are available from a wind-tunnel test campaign performed at the European Transonic Wind Tunnel in Cologne, Germany.
\end{abstract}

\section{Introduction}

$\mathbf{T}$ HE aerodynamic performance of an aircraft strongly depends on its wing design and aeroelastic characteristics. The accurate calculation of aerodynamic parameters is of significant importance during the design and analysis of an aircraft configuration. Over the last two decades, the field of Reynolds-averaged NavierStokes (RANS) based Computational Fluid Dynamics (CFD) has significantly progressed regarding robustness, efficiency, and the capability to handle complex configurations [1,2]. Today, incremental aerodynamic coefficients of typical transonic aircraft can be calculated with acceptable accuracy, both around the cruise design point and for nonseparated flows in general. However, regarding absolute values and increments at off-design conditions near the edges of the flight envelope, significant challenges still exist to accurately compute aerodynamic data and model the underlying flow physics.

Based on these challenges, a working group of the AIAA Applied Aerodynamics Technical Committee initiated the CFD Drag Prediction Workshop (DPW) series in 2001, resulting in six international workshops to date. Participants and committee results have been summarized in more than 120 papers [3-7]. DLR's Institute of Aerodynamics and Flow Technology is supporting DPW as a committee member and participant [ㅁ-12]. The second author is a DPW committee member and has been a participant in past DPW workshops [13-16].

Starting from DPW-4, the NASA Common Research Model $(\mathrm{CRM})^{\S}$ civil transport aircraft configuration (depicted in Fig. 1), designed by NASA's subsonic fixed-wing technical working group and by Vassberg et al. [17], has been used as the reference geometry.

Presented as Paper 2017 at the 55th AIAA Aerospace Sciences Meeting, Grapevine, TX, 9-13 January 2017; received 9 March 2017; revision received 18 May 2017; accepted for publication 18 May 2017; published online 30 June 2017. Copyright $\odot 2017$ by German Aerospace Center (DLR). Published by the American Institute of Aeronautics and Astronautics, Inc., with permission. All requests for copying and permission to reprint should be submitted to CCC at www.copyright.com; employ the ISSN 0021-8669 (print) or 1533-3868 (online) to initiate your request. See also AIAA Rights and Permissions www.aiaa.org/randp.

*Research Scientist, Department of Transport Aircraft, Institute for Aerodynamics and Flow Technology, Lilienthalplatz 7. Member AIAA.

${ }^{\dagger}$ Professor, Department of Mechanical Engineering, 1000 East University Avenue. Associate Fellow AIAA.

*Data available online at http://aiaa-dpw.larc.nasa.gov/ [retrieved 14 June 2017].

${ }^{\S}$ Data available online at http://commonresearchmodel.larc.nasa.gov/ [retrieved 14 June 2017].
Geometrical and experimental data of the model are found on the NASA CRM website (see footnote $\S$ ).

The sixth workshop had a focus on drag increment, grid adaptation, and, following observations from the fourth and fifth workshops, aeroelastic studies. Test cases include five series of computations. This paper summarizes the participants' results for the aeroelastic simulation to be performed in the optional test case 5 . The test case is focused on an investigation of static aeroelastic effects of the CRM wing/body (WB) configuration at $M_{\infty}=0.85$ and $C_{L}=0.5 \pm 0.0001$, where significant wing deformation is expected. CFD simulations are run on the medium baseline grid level and are coupled to a computational structural analysis model using a finite element model supplied by NASA and constructed using the commercially available structural analysis code NASTRAN. - The finite element model (FEM) is based on a detailed representation of the actual CRM wind-tunnel model and is illustrated in Fig. 2. The model is constructed with nacelles and pylons as separate components, which can be included or removed from the model as desired. The FEM model is constructed entirely of tetrahedral elements (total of approximately 6.8 million elements) and a detailed illustration for the case with removed nacelles and pylons (i.e., case 5 herein) is provided in Fig. 2. Please note that, although the test case uses the WB configuration, the structural model includes a horizontal tail as shown in the figure. However, because coupling between CFD and structural analysis is limited to wing deflections only, the presence of the tail plane has been considered negligible.

The FEM model can be used either directly with compatible structural solvers to compute the CRM wind-tunnel model structural response or can be used to generate modal shapes and frequencies (eigenmodes and eigenvectors) for use in a simplified modal analysis. For this purpose, the DPW committee has computed the first 30 modal shapes and frequencies based on the FEM model with nacelles removed. The modal information was computed using the Lanczos algorithm in the Abaqus commercial software. The first eight mode shapes are depicted in Fig. 3, and the frequencies for these modes ranges from 39.36 to $128.37 \mathrm{~Hz}$ for the first and eighth mode, respectively. The finite element model and the first 30 mode shapes and eigenfrequencies are available via the CRM website (see footnote $\$$ ).

Static aeroelastic wing deflections are calculated, starting from the undeformed geometry. Computed results of lift, drag, pitching

IData available online at http://aiaa-dpw.larc.nasa.gov/Workshop6/ DPW6_Test_Cases_2016-04-21.pdf [retrieved 14 June 2017].

**Data available online at https://www.mscsoftware.com/product/ msc-nastran/ [retrieved 14 June 2017]. 


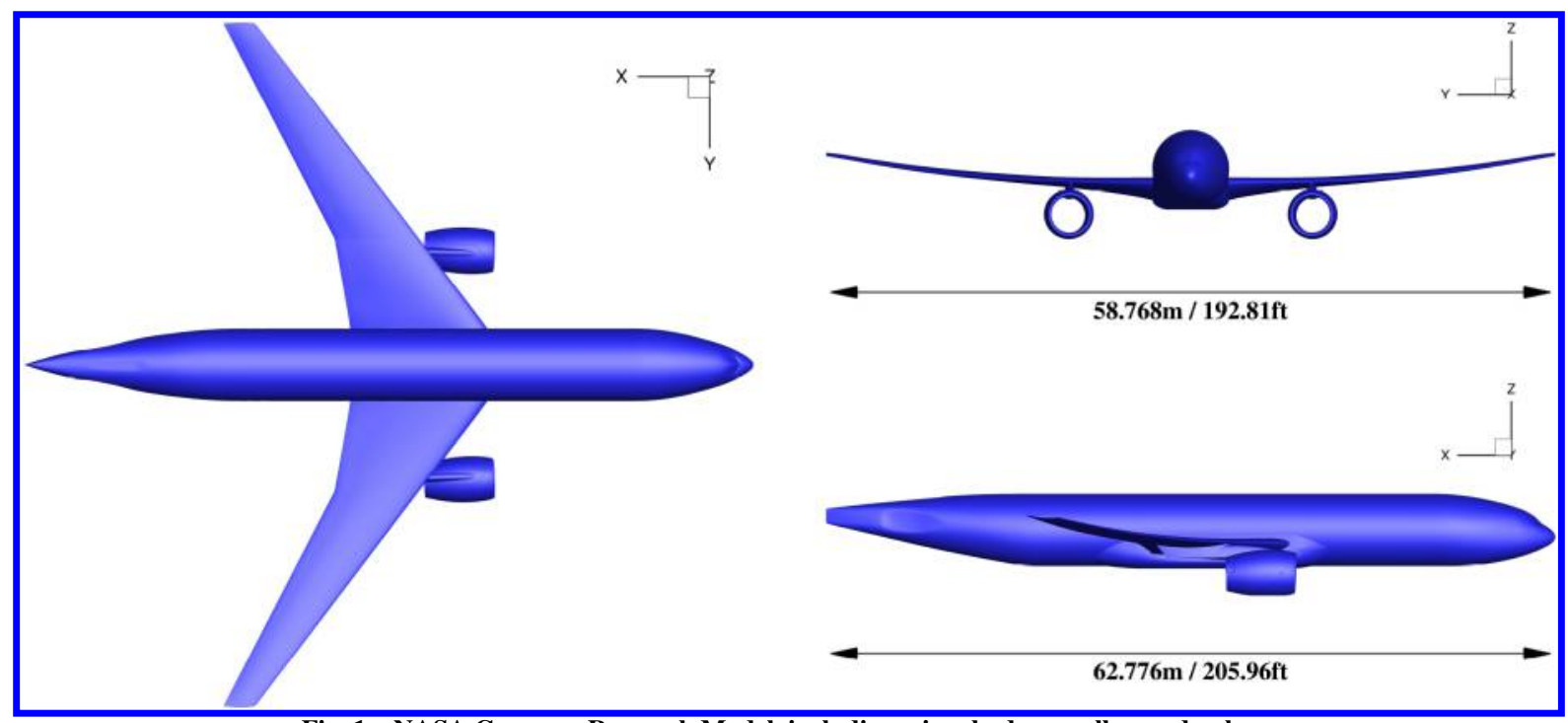

Fig. 1 NASA Common Research Model, including wing, body, nacelles, and pylons.

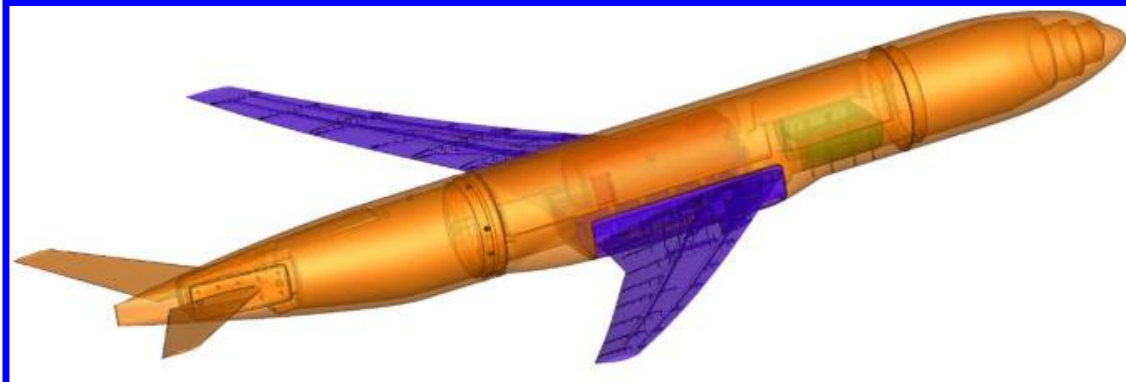

a) Overall configuration

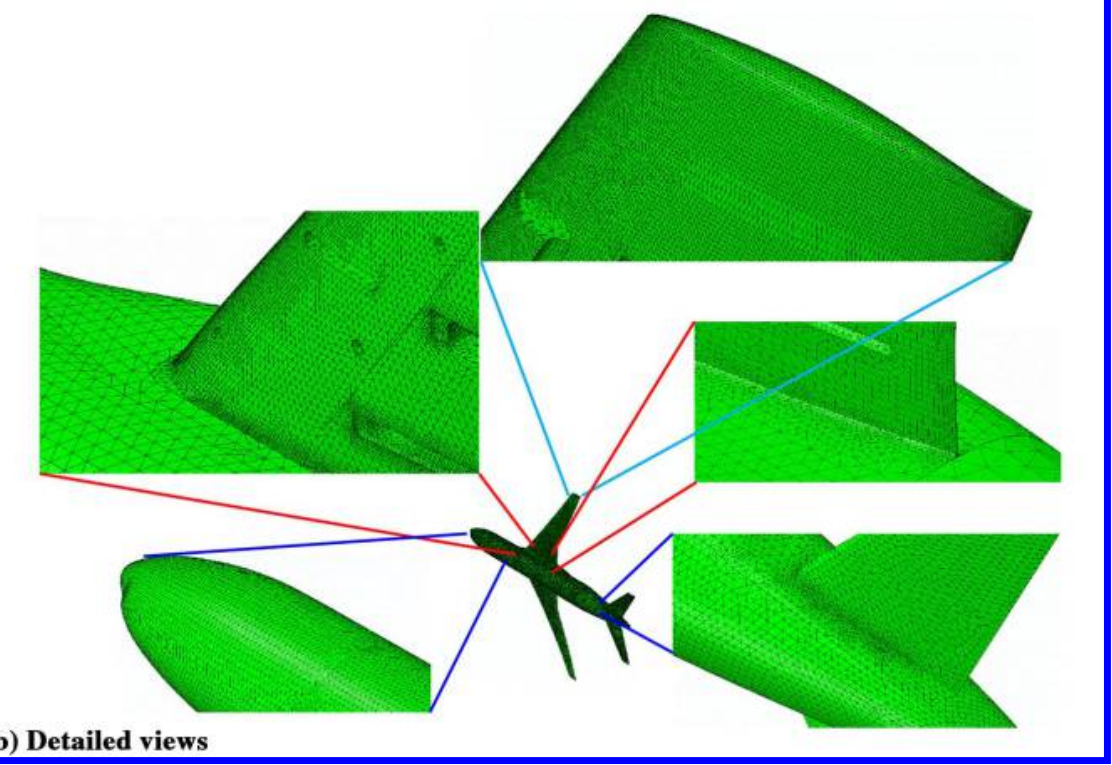

Fig. 2 Illustration of structural model for CRM wind-tunnel model.

moment, sectional lift and pitching moment, wing section static pressure and skin friction coefficients at specified spanwise locations, locations of flow separations on the wing and side of body, and wing bending and twist deformation were requested. A total of four data sets were provided for case 5 . The participating teams are listed in Table 1 .

\section{Numerical Simulations}

This section gives an overview of the numerical methods used by the participants for CFD, computational structural mechanics (CSM), interpolation of aerodynamic loads, and mesh deformation. A typical example for a static aeroelastic simulation approach is shown in Fig. 4 for illustration purposes.

Case 5 was optional, and data were submitted by four teams, out of a total of 25 participating teams in the DPW-6 workshop [18]. Table 2 lists the main features of the individual aeroelastic simulation approaches used by the participants. The data set keys were defined by Tinoco et al. in [18].

Regarding the basic fluid-structure interaction (FSI) method, three participants (J4, L2, and T1) apply a linear static coupling approach, where the structural system equations are solved within each coupling 


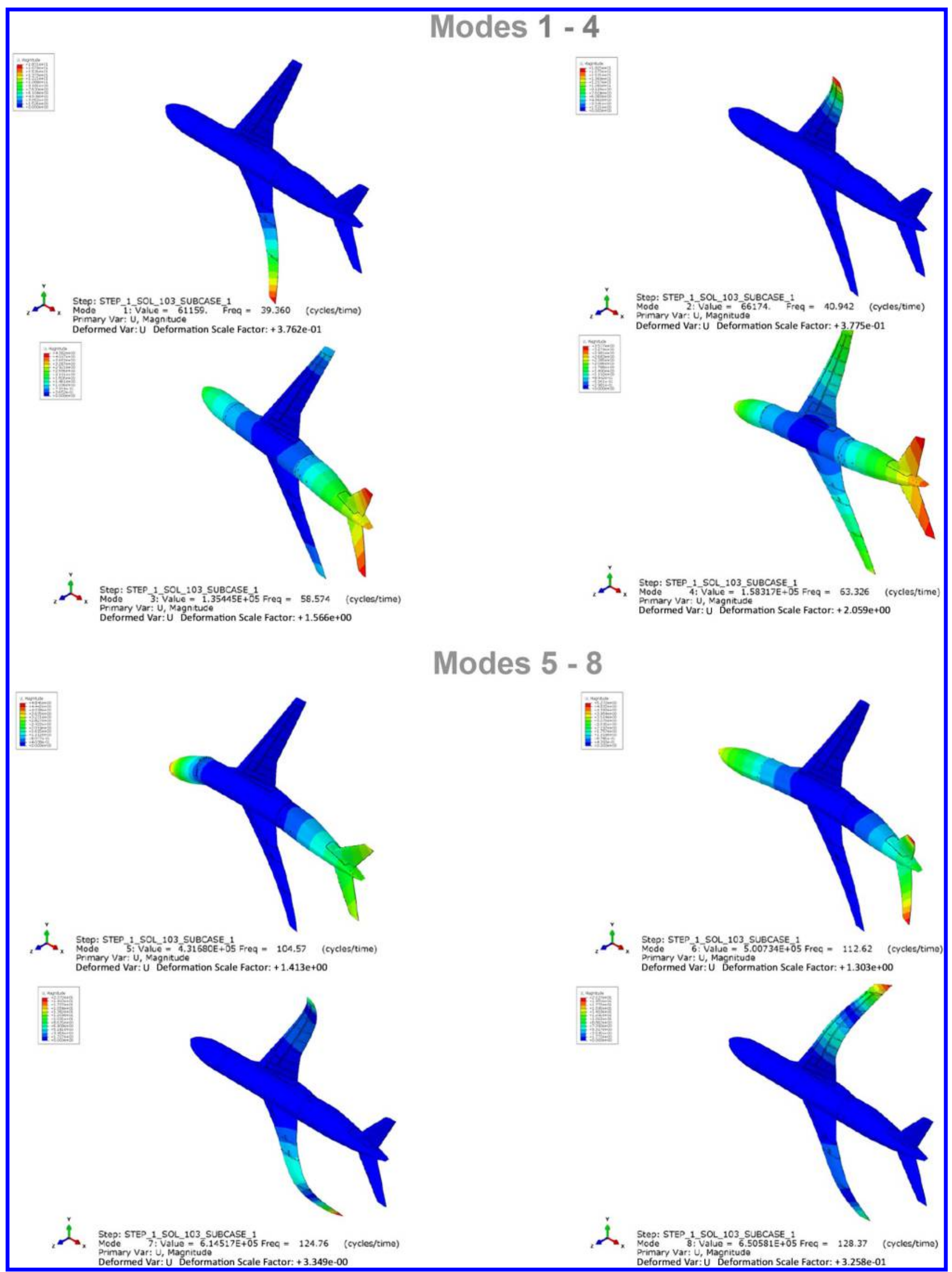

Fig. 3 Illustration of first eight modes for CRM structural model with removed nacelles.

loop. Team V5 uses a linear modal approach. Here, a set of normal modes is computed before starting the actual FSI simulation, and the static deflections are assembled through a superposition of modes in each coupling loop. All participants use different finite volume flow solvers and turbulence models. T1 uses the common multiblock CFD mesh, all others use custom unstructured (J4, L2) or structured (V5) 
Table 1 Test case 5 participants

\begin{tabular}{lcccc}
\hline \hline Team & Data set key & Organization & Country & Type \\
\hline 9 & J4 & Metacomp & United States & Commercial \\
& & Technologies & & \\
11 & L2 & DLR e.V. & Germany & Government \\
19 & T1 & CARDC & China & Government \\
21 & V5 & Embraer S.A. & Brazil & Industry \\
\hline \hline
\end{tabular}

meshes. Interpolation of aerodynamic forces between the CFD surface mesh and the structural model surface is performed using a nearestneighbor search algorithm (teams J4, L2, and V5) or a thin-plate spline (team T1). The model suspension in the structural analysis is located at the intersection line between wing root and belly fairing for teams $\mathrm{J} 4$ and $\mathrm{T} 1$ and at the balance interface for team L2. The boundary conditions in the modal analysis (team V5) are equivalent to a suspension at the model's center of gravity. Mesh deformation algorithms used include radial basis functions (RBF, teams J4, L2, and T1) and linear torsional springs analogy (LTSA, team V5).

Unfortunately, the NASTRAN bulk data file provided on the CRM website (see footnote §) did not include any definition of boundary conditions for the system matrices, leaving the structural analysis problem statically undetermined. As a result, the teams used differing locations for the model suspension (cf. Table 2). The correct suspension (i.e., the one corresponding to the actual model attachment in the wind tunnel) is inside the fuselage at the inner cylindrical surface of the balance interface. At this location, the model is mounted to the internal wind-tunnel balance during the test. With the wing root suspension used by two participants, zero deflection is prescribed along the junction line between wing and belly fairing. This suspension results in neglecting the compliancy of the structural components located between balance interface and wing root, leading to smaller deformations. Generally, the different model suspensions used in the structural analysis cause minor differences in the computed wing deformations, as will be discussed later on.

\section{A. Flow Solvers}

\section{TAU}

The Reynolds-averaged Navier-Stokes flow solver TAU [19] has been developed at DLR starting in the mid 1990s. The code originates from the German CFD project MEGAFLOW [19-21], which has integrated developments of DLR, aircraft industry, and universities.

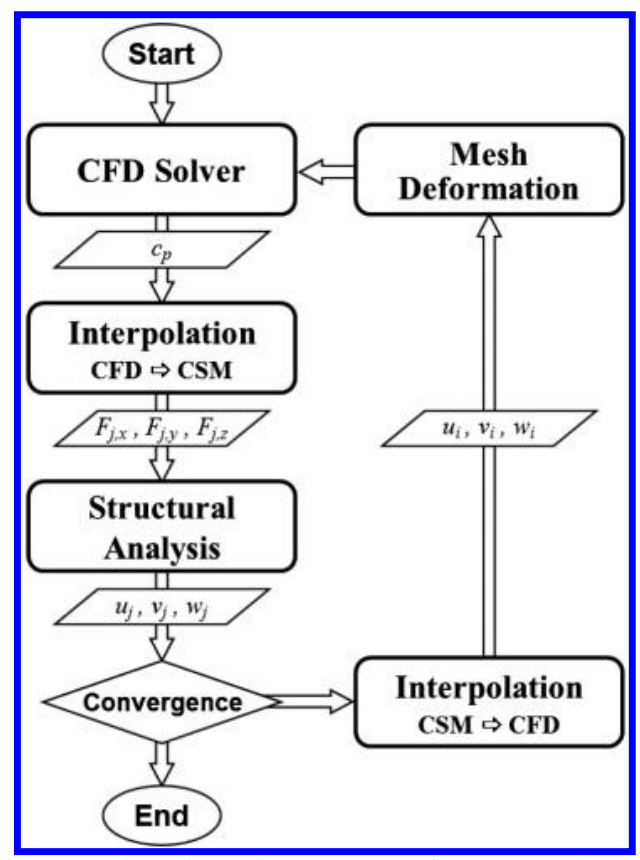

Fig. 4 Numerical simulation procedure for aeroelastic analyses.
Today, the software package is continuously upgraded and expanded by the institute's Center for Computer Applications in AeroSpace Science and Engineering department and is used by DLR and European partners in industry, research, and academia.

TAU is an edge-based finite volume unstructured solver that uses the dual grid technique and fully exploits the advantages of hybrid grids. The numerical scheme is based on the finite volume method and provides different spatial discretization schemes, like upwind and central [19]. The central scheme is of second-order accuracy and employs the Jameson-type of artificial dissipation in scalar and matrix mode $[22,23]$. Time integration is performed using both the explicit Runge-Kutta multistage and the lower-upper symmetric Gauss-Seidel (LU-SGS) schemes. TAU has been developed with a particular focus on industrial aeronautical applications, thus providing techniques like overlapping grids for treating unsteady phenomena and complex geometries. A detailed description of TAU is provided in [19].

For DPW-6, TAU was used to compute steady RANS solutions with LU-SGS time integration, central spatial discretization, and the new matrix dissipation formulation available in release 2015.2.0. The negative version of the Spalart-Allmaras turbulence model (SA-neg) [24] was used for these calculations.

\section{CMSoft, Inc. AERO-F}

AERO-F is a three-dimensional compressible Euler/NavierStokes arbitrary Lagrangian Eulerian finite volume flow solver. It features state-of-the-art RANS and detached- and large-eddy simulation turbulence models. It operates on unstructured hybrid meshes that can be static, rigidly moved in time by a flow-driven motion of a six-degrees-of-freedom rigid body, or deformed and possibly reconfigured by a movable surface. These can also be simultaneously moved and deformed in time by a prescribed surface motion and/or deformation or a coupling with the dynamics of a flexible structure. AERO-F can also perform linearized simulations for rapidly predicting the response of a flow to perturbations and compute the sensitivity of output results to variations of shape and flow parameters. The Spalart-Allmaras turbulence model with quadratic constitutive relation (QCR) modification (SA-QCR2013) [25] was used in these calculations.

\section{TRIP}

The TRIP CFD code has been developed at the China Aerodynamic Research and Development Center (CARDC). TRIP solves the RANS equations using a discretization based on a finite volume approach for multiblock structured meshes. The computations done for this case made use of the 2003 version of the shear stress transport (SST) turbulence model (SST-2003) [26].

\section{4. $\mathrm{CFD++}$}

CFD++ is a commercially available RANS CFD solver distributed by Metacomp Technologies, Inc. CFD++ makes use of a unified unstructured higher-order total variation diminishing interpolation convection scheme. A positivity-preserving Riemann-solver-based flux computation is used, and both cell and vertex-based polynomial reconstruction are available. CFD++ makes use of an algebraic multigrid agglomeration linear solver for enhanced convergence to steady state. The realizable $k-\epsilon$ turbulence model was used for these calculations.

\section{B. Structural Solver}

Although the structural model of the CRM wind-tunnel model was built using NASTRAN and provided as part of the workshop, participants were free to use their method of choice for computing the structural response of this model. Of the four participants, three used a linear finite element approach and one used a linear modal approach. Data set L2, using the TAU CFD code, employed the commercially available NASTRAN code to compute the structural response. Linear, static analyses were performed using NASTRAN's solution sequence 101 . The model suspension boundary condition was defined at the balance interface. 
Table 2 Overview of aeroelastic simulation methods used for test case 5

\begin{tabular}{lcccc}
\hline \hline Data set key & J4 & L2 & T1 & V5 \\
\hline Structural modeling & Linear static & Linear static & Linear static & Linear modal \\
Flow solver & Finite volume & Finite volume & Finite volume & Finite volume \\
Solver name & CFD++ & TAU & TRIP & CMS AERO-F \\
Turbulence model & Realizable $k-\varepsilon$ & SA-neg & SST-2003 & SA-QCR2013 \\
Model suspension & Wing root & Balance interface & Wing root & Center of gravity \\
Force interpolation & Nearest neighbor & Nearest neighbor & Thin-plate spline & Nearest neighbor \\
Mesh deformation & RBF & RBF & RBF & LTSA \\
\hline \hline
\end{tabular}

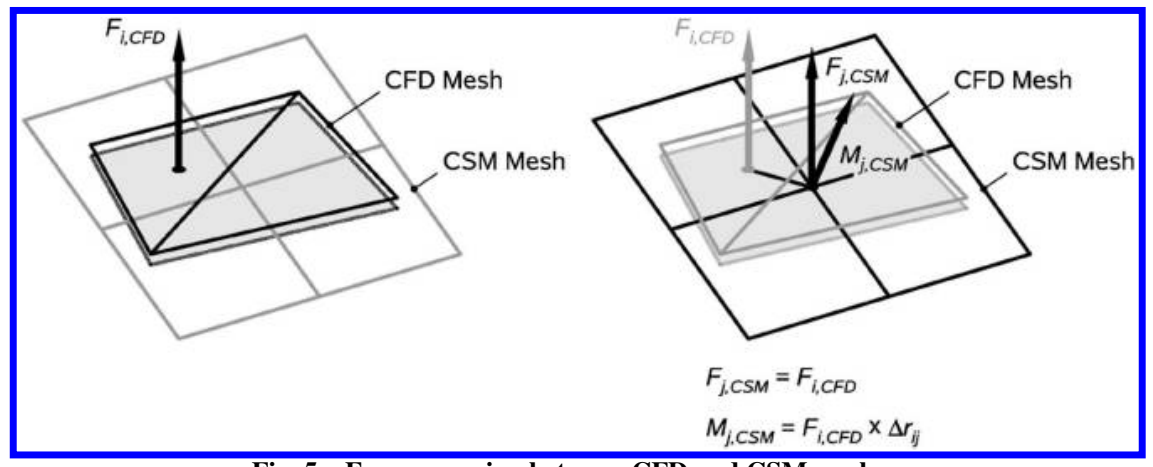

Fig. 5 Force mapping between CFD and CSM meshes.

Data set J4 (using CFD++) employed the CSM + + structural analysis software distributed by Metacomp Technologies, Inc. $\mathrm{CSM}++$ is a finite-element-based structural solver that can be used to perform static, transient, and eigenmode analyses. The model suspension boundary condition was defined at the wing root.

Data set V5 (using CMSoft, Inc. (CMS) AERO-F) employed a linear modal analysis. In this case, the mode shapes and frequencies were not taken directly from those provided by the DPW committee, but rather were computed independently using NASTRAN assuming a linear structure. The first 10 modes were used in the structural analysis computations. The model suspension boundary condition was defined at the center of gravity of the model in this case.

Data set T1 (using TRIP) performed a reduction of the stiffness matrix for the structural model and computed the structural response outside of NASTRAN using a linear static finite element structural solver. The model suspension boundary condition was defined at the wing root for this case.

\section{Force Interpolation Methods}

1. Nearest-Neighbor Search

Nearest-neighbor search algorithms enable a conservative interpolation of aerodynamic forces between meshes with largely varying spatial resolution differences [27] or when connectivity data of the finite element surface nodes are not available. For a given CFD face centroid $i$, the nearest neighboring CSM grid point $j$ is identified and a force component $F_{j, \text { CSM }}$ and associated moment $M_{j, \text { CSM }}=F_{i, \text { CFD }} \times \Delta r_{i j}$ are mapped to node $j$, as shown in Fig. 5. Alternatively, the force and moment values can be projected onto the basis functions of the closest FEM surface element. In both cases, the transpose of the CFD to CSM interpolation matrix is used to propagate the structural deflections back to the CFD surface mesh, thus ensuring the principle of conservation of virtual work.

\section{Thin-Plate Spline}

Data set $\mathrm{T} 1$ used the thin-plate spline approach for interpolating forces from the CFD surface to the structural model and for returning displacements from the structural model to the CFD mesh. In this approach, the structural model is idealized as a planar surface and displacements are projected onto the CFD surface mesh using a twodimensional spline formulation.

\section{Mesh Deformation Algorithms}

Various approaches for deforming the CFD mesh in response to the displacements generated by the structural model have been developed in the literature. These include spring analogy methods, linear elastic analogies, and interpolation methods, such as those based on radial basis functions. Three participants employed the latter radial basis function approach. This technique is appealing because it does not require any knowledge of the CFD grid connectivity and scales only as a function of the number of surface mesh points. The method has been shown to produce smooth deformed meshes that are resistant to the production of negative volume cells. However, the radial basis function approach results in a dense matrix system to be solved, which can become costly for very fine surface meshes. The straightforward spring analogy method is well known to be less robust than alternative approaches, such as linear elastic and radial basis function methods. Data set V5 employs an augmented approach that makes use of torsional springs, which substantially increases the robustness of this approach.

\section{Results}

In Fig. 6, the chordwise distribution of static pressure coefficient is plotted. Four spanwise sections between $\eta=0.131$ and $\eta=0.950$ were selected for comparison. Sectional static pressure data have been submitted by teams J4, L2, and T1. Experimental validation data have been taken from a test campaign performed at the European Transonic Wind Tunnel (ETW) in Cologne, Germany, as part of the European research project European Strategic Wind Tunnels Improved Research Potential.it

A generally good agreement is found between the different participants and measured data. With all three teams, shock location is predicted somewhat downstream of the experiment. Shock location for teams $\mathrm{J} 4$ and T1 is predicted more upstream compared with L2. This is believed to be due to the one-equation turbulence model used by L2, whereas J4 and T1 have used a two-equation model. Differences observed in the pressure distributions on the lower wing surface are very small, indicating that only minor deviations in local angle of attack (i.e., spanwise wing twist distribution) occur. In summary, the aeroelastic effects appear to be correctly captured by all participants.

Figures $7 \mathrm{a}$ and $7 \mathrm{~b}$ show the spanwise wing bending and twist distributions, repsectively. Numerical results are compared with ETW

${ }^{\dagger}$ Data available online at http://www.eswirp.aero/ [retrieved 14 June $2017]$. 

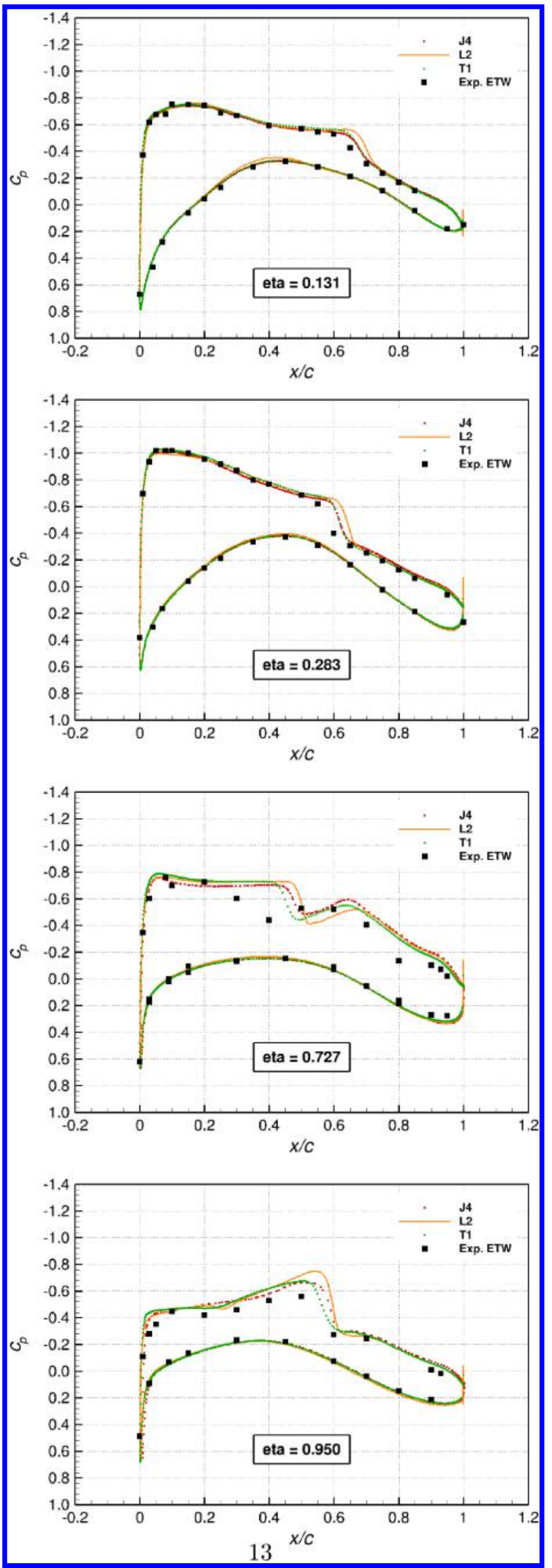

Fig. 6 Wing static pressure distributions.

deformation data from run no. 182, data point no. $531(\alpha=2.99 \mathrm{deg}$, $\left.C_{L}=0.514\right)$. Bending deflections are given in millimeters and refer to the $0.027 \%$ scale wind-tunnel model. For bending, the best agreement is found for team V5. Here, the maximum deflection deviation at wing

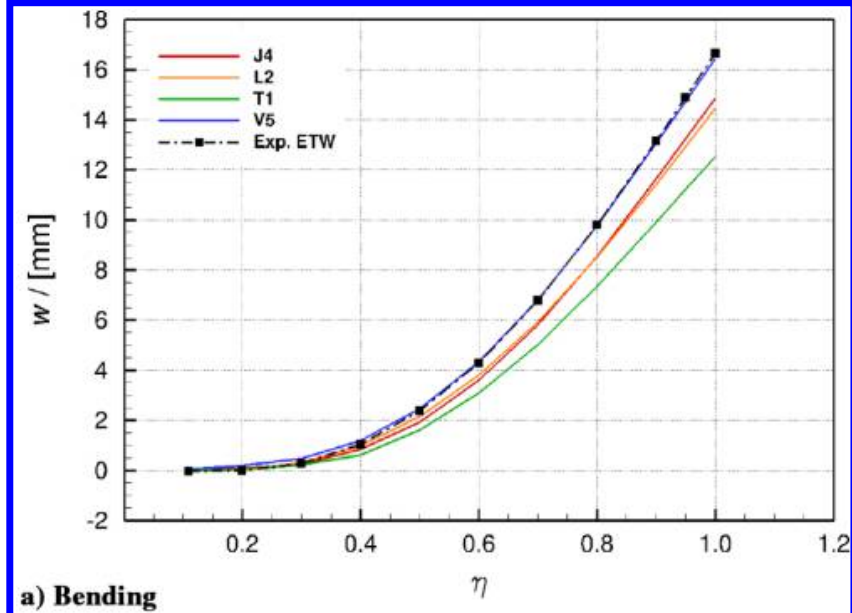

a) Bending

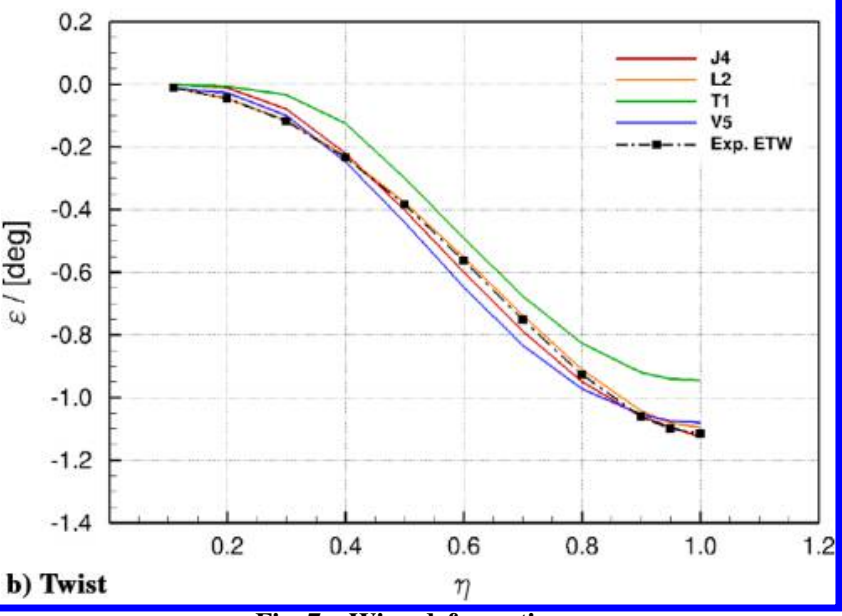

Fig. 7 Wing deformations.

tip is $\Delta w=-0.19 \mathrm{~mm}$, followed by teams $\mathrm{J} 4(\Delta w=-1.80 \mathrm{~mm})$ and L2 $(\Delta w=-2.18 \mathrm{~mm})$. The largest deviation occurs for team T1 at $\Delta w=-4.11 \mathrm{~mm}$. It is assumed that the deviations between the four teams are caused by a combination of differences in the wing's sectional and spanwise loadings, indicated by the static pressure distributions in Fig. 6, which increase toward the outboard wing sections, and the structural analysis methods used. Apart from that, the deflection curve progression over span is very close to measured data for all participants. This also indicates that the spanwise lift distributions between the different teams match very well. However, no spanwise lift data are available to support this assumption.

The aerodynamically more relevant twist deformation is plotted in Fig. $7 \mathrm{~b}$. Here, the differences between the finite element model suspensions become more apparent than for bending. For teams J4 and $\mathrm{T} 1$, who used the model suspension at the wing root, the twist deflection and spanwise deflection gradient at the innermost wing station are very close to zero, whereas the measurements show a small deflection and nonzero gradient caused by the compliance of the wind-tunnel model structure. The wing root model suspension affects twist on the inboard wing up to approximately $30-40 \%$ span. Twist deflection results for participants T1 and V5 show a steeper gradient over most of the span compared with the experimental data. It is assumed that these differences are caused by differences in the structural analysis methods used (cf. Sec. II.B). Unfortunately, no sectional lift and moment data, which would enable a more detailed deformation result analysis, have been submitted by the participants.

The results are consistent with similar static aeroelastic results produced by participants at the first Aeroelastic Prediction Workshop, which focused on dynamic aeroelastic problems, but also included a static aeroelastic test case [28]. However, in this first Aeroelastic Prediction Workshop, computations were performed using linear modal analysis because the modes were interpolated 


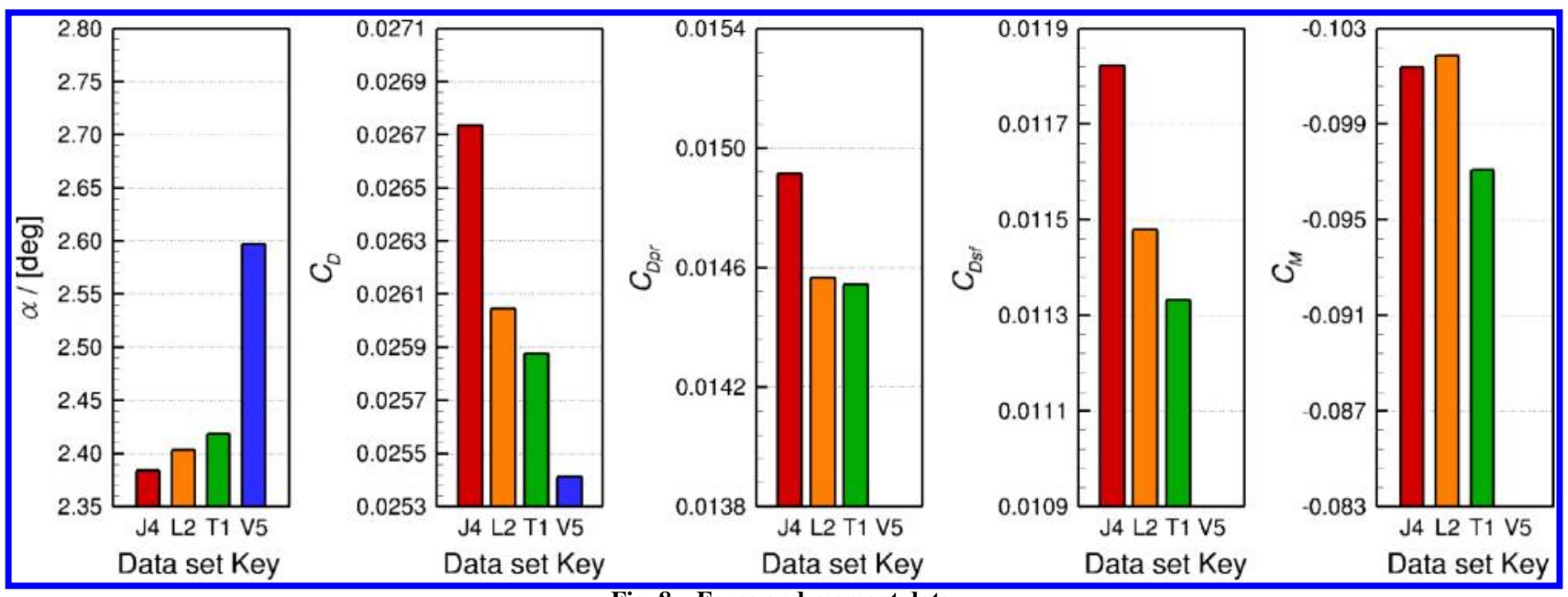

Fig. 8 Force and moment data.

directly onto the workshop CFD surface meshes and provided as such by the committee, for ease of use.

Figure 8 depicts the computed lift, drag, and pitching moment, as well as the incidence obtained with the prescribed $C_{L}$ condition for the four aeroelastic data sets. The vertical axis minimum and maximum values in each subfigure indicate the ranges of the aggregate results obtained in case 2. Keeping in mind that these results are obtained on meshes representative of the medium workshop meshes, the mean and the spread of the results compare relatively well with the aggregate results obtained using the predetermined aeroelastically deflected grids used in case 2 for the workshop. Of these, only the pitching moments for two data sets (out of only three provided) appear to be at the edge of the range (more negative) of those reported in case 2. Given the range of grid types and turbulence models used in this sparse data set, it should not be surprising that the spread is slightly larger when the additional effect of aeroelastic deflection is included in the simulations. To draw meaningful conclusions, a larger data set involving more participants including subsets using the same turbulence models and grid types would be desirable.

\section{Conclusions}

A generally good agreement was found between the teams participating in test case 5. Deviations from the experimental data observed for chordwise shock location were on the order of magnitude expected from medium-density grids. Additional influence factors likely are differences in wing twist distribution between the individual participants and the different turbulence models used. Some discrepancies were also found for the slope of the spanwise twist curve. At the wing root, these were traced back to different boundary conditions applied to the structural model. Other deviations from the experimental data are assumed to be caused by differences in the structural analysis methods used by the participants. Although aeroelastic simulations are increasing in importance, the fact that only 4 out of 25 participants chose to perform the optional case 5 in DPW-6 indicates that coupled static aeroelastic computations remain the exception rather than the norm in the current state of the practice.

\section{Acknowledgments}

Experimental data from the European Transonic Wind Tunnel were made available through the European research project European Strategic Wind Tunnels Improved Research Potential. The authors wish to thank Melissa B. Rivers, Research Engineer at the Configuration Aerodynamics Branch at NASA Langley Research Center and the Drag Prediction Workshop Organizing Committee for the excellent collaboration.

\section{References}

[1] Becker, K., and Vassberg, J., "Numerical Aerodynamics in Transport Aircraft Design," Notes on Numerical Fluid Mechanics and Multidisciplinary Design, edited by E.-H. Hirschel, and E. Krause, Vol. 100, Springer-Verlag, Berlin, 2009, pp. 209-220.

[2] Rossow, C.-C., and Cambier, L., "European Numerical Aerodynamics Simulation Systems," Notes on Numerical Fluid Mechanics and Multidisciplinary Design, edited by E.-H. Hirschel, and E. Krause, Vol. 100, Springer-Verlag, Berlin, 2009, pp. 189-208.

[3] Levy, D., Zickuhr, T., Vassberg, J., Agrawal, S., Wahls, R., Pirzadeh, S., and Hemsch, M., "Summary of Data from the First AIAA CFD Drag Prediction Workshop," AIAA Paper 2002-0841, Jan. 2002.

[4] Laflin, K., Klausmeyer, S., Zickuhr, T., Vassberg, J., Wahls, R.,

Morrison, J., Brodersen, O., Rakowitz, M., Tinoco, E., and Godard, J.-L., "Data Summary from Second AIAA Computational Fluid Dynamics Drag Prediction Workshop," Journal of Aircraft, Vol. 42, No. 5, 2005, pp. $1165-1178$. doi:10.2514/1.10771

[5] Vassberg, J., Tinoco, E., Mani, M., Brodersen, O., Eisfeld, B., Wahls, R., Morrison, J., Zickuhr, T., Laflin, K., and Mavriplis, D., "Abridged Summary of the Third AIAA Computational Fluid Dynamics Drag Prediction Workshop," Journal of Aircraft, Vol. 45, No. 3, 2008, pp. 781-798. doi: $10.2514 / 1.30572$

[6] Vassberg, J., Tinoco, E., Mani, M., Zickuhr, T., Levy, D., Brodersen, O., Crippa, S., Wahls, R., Morrison, J., Mavriplis, D., and Murayama, M., "Summary of the Fourth AIAA Drag Prediction Workshop," AIAA Paper 2010-4547, June 2010.

[7] Levy, D., Laflin, K., Tinoco, E., Vassberg, J., Mani, M., Rider, B., Rumsey, C., Wahls, R., Morrison, J., Brodersen, O., Crippa, S., Mavriplis, D., and Murayama, M., "Summary of Data from the Fifth AIAA CFD Drag Prediction Workshop," AIAA Paper 2013-0046, Jan. 2013.

[8] Rakowitz, M., Sutcliffe, M., Eisfeld, B., Schwamborn, D., Bleeke, H., and Fassbender, J., "Structured and Unstructured Computations on the DLR-F4 Wing-Body Configuration," AIAA Paper 2002-0837, 2002.

[9] Brodersen, O., Rakowitz, M., Amant, S., Larrieu, P., Destarac, D., and Sutcliffe, M., "Airbus, ONERA, and DLR Results from the Second AIAA Drag Prediction Workshop," Journal of Aircraft, Vol. 42, No. 4, 2005, pp. $932-940$. doi: $10.2514 / 1.8662$

[10] Brodersen, O., Eisfeld, B., Raddatz, J., and Frohnapfel, P., "DLR Results from the Third AIAA CFD Drag Prediction Workshop," Journal of Aircraft, Vol. 45, No. 3, 2008, pp. 823-836. doi: $10.2514 / 1.30628$

[11] Brodersen, O., Crippa, S., Eisfeld, B., Keye, S., and Geisbauer, S., "DLR Results from the Fourth AIAA CFD Drag Prediction Workshop," AIAA Paper 2010-4223, June 2010

[12] Brodersen, O., and Crippa, S., "RANS-Based Aerodynamic Drag and Pitching Moment Predictions for the Common Research Model," DGLR STAB Workshop 2012, New Results in Numerical and Experimental Fluid Mechanics IX Notes on Numerical Fluid Mechanics and Multidisciplinary Design, Vol. 124, Springer-Verlag, 2014, pp. 485-493.

[13] Mavriplis, D. J., and Levy, D. W., "Transonic Drag Prediction Using an Unstructured Multigrid Solver," Journal of Aircraft, Vol. 42, No. 4, 
2005, pp. 887-893. doi: $10.2514 / 1.8233$

[14] Mavriplis, D. J., "Third Drag Prediction Workshop Results Using the NSU3D Unstructured Mesh Solver," Journal of Aircraft, Vol. 45, No. 3 , May 2008, pp. 750-761. doi: $10.2514 / 1.29828$

[15] Mavriplis, D. J., and Long, M., "NSU3D Results from the Fourth AIAA CFD Drag Prediction Workshop," AIAA Paper 2010-4364, June 2010.

[16] Mavriplis, D. J., Vassberg, J. C., Tinoco, E. N., Mani, M., Brodersen, O. P.,

-Eisfeld, B., Wahls, R. A., Morrison, J. H., Zickuhr, T., and Levy, D., "Grid Quality and Resolution Issues from the Drag Prediction Workshop Series," Journal of Aircraft, Vol. 46, No. 3, 2009, pp. 935-950. doi:10.2514/1.39201

[17] Vassberg, J., DeHaan, M., Rivers, S., and Wahls, R., "Development of a Common Research Model for Applied CFD Validation Studies," AIAA Paper 2008-6919, June 2008.

[18] Tinoco, E. N., Brodersen, O. P., Keye, S., Laflin, K. R., Feltrop, E., Vassberg, J. C., Mani, M., Rider, B., Wahls, R. A., Morrison, J. H., Hue, D., Gariepy, M., Roy, C. J., Mavriplis, D. J., and Murayama, M., "Summary of Data from the Sixth AIAA CFD Drag Prediction Workshop: CRM Cases 2 to 5," AIAA Paper 2017-1208, Jan. 2017.

[19] Gerhold, T., "Overview of the Hybrid RANS Code TAU," MEGAFLOW, edited by N. Kroll, and J. Fassbender, Vol. 89, Notes on Numerical Fluid Mechanics and Multidisciplinary Design, SpringerVerlag, Berlin, 2005, pp. 81-92.

[20] Galle, M., "Ein Verfahren zur numerischen Simulation kompressibler, reibungsbehafteter Strömungen auf hybriden Netzen," Ph.D. Thesis, Univ. Stuttgart, Stuttgart, Germany, 1999.
[21] Kroll, N., Rossow, C.-C., Becker, K., and Thiele, F., "MEGAFLOW-A Numerical Flow Simulation System," Aerospace Science Technology, Vol. 4, No. 4, 2000, pp. 223-237. doi:10.1016/S1270-9638(00)00131-0

[22] Jameson, A., Schmidt, W., and Turkel, E., "Numerical Solution of the Euler Equations by Finite Volume Methods Using Runge-Kutta Time Stepping Schemes," AIAA Paper 1981-1259, Jan. 1981.

[23] Swanson, R. C., and Turkel, E., "On Central Differences and Upwind Schemes," Journal of Computational Physics, Vol. 101, No. 2, 1992, pp. 292-306. doi:10.1016/0021-9991(92)90007-L

[24] Allmaras, S. R., Johnson, F. T., and Spalart, P. R., "Modifications and Clarifications for the Implementation of the Spalart-Allmaras Turbulence Model," Seventh International Conference on Computational Fluid Dynamics (ICCFD7), ICCFD7-1902, Big Island, HI, July 2012.

[25] Mani, M., Babcock, D., Winkler, C., and Spalart, P., "Predictions of a Supersonic Turbulent Flow in a Square Duct," AIAA Paper 2013-0860, 2013.

[26] Menter, F., Kuntz, M., and Langtry, R., "Ten Years of Industrial Experience with the SST Turbulence Model," Turbulence, Heat and Mass Transfer, Vol. 4, No. 1, 2003, pp. 625-632.

[27] Heinrich, R., Wild, J., Streit, T., and Nagel, B., "Steady Fluid-Structure Coupling for Transport Aircraft," ONERA-DLR Aerospace Symposium, Oct. 2006

[28] Heeg, J., Chwalowski, P., Florance, J. P., Wieseman, C. D., Schuster, D. M., and Perry, R. B., "Analysis of Test Case Computations and Experiments for the Aeroelastic Prediction Workshop," AIAA Paper 2013-0783, Jan. 2013. 